Теорія Ймовір. та Матем. Статист. Вип. 81, 2010
Theor. Probability and Math. Statist.

No. 81, 2010, Pages 101-115

S 0094-9000(2011)00813-7

Article electronically published on January 20, 2011

\title{
ASYMPTOTIC BEHAVIOR OF THE DISTRIBUTION OF THE MAXIMUM OF A CHENTSOV FIELD ON POLYGONAL LINES
}

UDC 519.21

\author{
N. V. KRUGLOVA
}

\begin{abstract}
Let $X(s, t)$ be a two-parameter Chentsov field. The asymptotic behavior of the tail of the distribution of the maximum of the field $X(s, t)$ on a polygonal line with several linear sections is found in the paper.
\end{abstract}

\section{INTRODUCTION}

We consider a two-parameter Chentsov field $X(s, t)$ (also known as the Brownian sheet). The definition of such a field was given in 1955 by Chentsov 1 in terms of densities of the distribution of the field $X(s, t)$. We use an equivalent definition due to Yeh $[9$. Let $D=[0,1] \times[0,1]$.

Definition 1.1. A real separable Gaussian random field $\{X(s, t):(s, t) \in D\}$ is called a Chentsov field if

1) $X(0, t)=X(s, 0)=0$ for all $s, t \in[0,1]$;

2) $\mathrm{E}[X(s, t)]=0$ for all $(s, t) \in D$;

3) $\mathrm{E}\left[X(s, t) X\left(s_{1}, t_{1}\right)\right]=\min \left\{s, s_{1}\right\} \min \left\{t, t_{1}\right\}$ for all $(s, t) \in D$ and $\left(s_{1}, t_{1}\right) \in D$.

The closed form of the distribution of functionals such as $\max _{(s, t) \in D} X(s, t)$ is still unknown for Chentsov fields. The distribution of the supremum of $X(s, t)$ on the boundary of the unit square is found by Paranjape and Park [7]. Klesov [4 found an explicit form of the probability

$$
P(L, g)=\mathrm{P}\left\{\sup _{(s, t) \in L} X(s, t)-g(s, t)<0\right\},
$$

where $X$ is a Chentsov field on $D, L$ is a polygonal line with two linear sections, and $g$ is a linear function.

The probabilities

$$
P(L, \lambda)=\mathrm{P}\left\{\sup _{(s, t) \in L} X(s, t)>\lambda\right\}
$$

are considered in [5] and [6], where $L$ is a polygonal line with several linear sections. In [5] and [6], the probability (2) is expressed in terms of the standard Gaussian distribution function in a rather complicated form; thus estimates for (2) are also of a considerable interest.

2010 Mathematics Subject Classification. Primary 60G15, 60G60.

Key words and phrases. Chentsov field, distribution of the maximum, Gaussian processes. 
Nice lower estimates for the distribution of the maximum of the Chentsov field on a square are obtained in [3]. Estimates of the distribution of the maximum of the Chentsov field on a unit square are considered in [2] in a neighborhood of the origin. Estimates in [2] imply that there is no clear-cut notion of a two-parameter reflection principle.

The main aim of this paper is to find the exact asymptotic behavior of probabilities (2) as $\lambda \rightarrow \infty$.

\section{Auxiliary Results}

2.1. The probability of reaching a level by the Chentsov field. Let $L$ be the polygonal line depicted in Figure 1. It has two linear sections with a unique common point $\left(x_{1}, y_{1}\right), 0<x_{1}<1,0<y_{1}<1$. The line $L$ can be conveniently treated as the set of points in $D$ such that

$$
L=\left\{(s, t): s a^{-1}+t=1, s \leq k ; s+t b^{-1}=1, s>k,(s, t) \in D\right\},
$$

where $a=x_{1} /\left(1-y_{1}\right), b=y_{1} /\left(1-x_{1}\right)$, and $k=a(b-1) /(a b-1)$ for $a, b>1$.

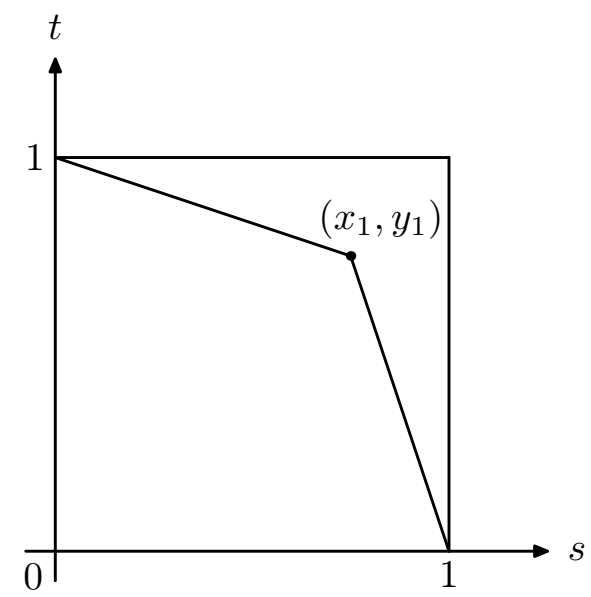

Figure 1. Polygonal line with two linear sections

In what follows we use the notation

$$
\Phi(x)=\frac{1}{\sqrt{2 \pi}} \int_{-\infty}^{x} e^{-t^{2} / 2} d t, \quad \Psi(x)=1-\Phi(x) .
$$

Let $\Gamma(z)=\int_{0}^{\infty} t^{z-1} e^{-t} d t, \operatorname{Re} z>0$, be the gamma function.

Theorem 2.1 (Paranjape and Park [7]). Let L be the polygonal line defined by (3). Let $X(s, t),(s, t) \in D$, be a Chentsov field. Then

$$
\begin{aligned}
P\left\{\sup _{(s, t) \in L} X(s, t) \leq \lambda\right\}= & \Phi\left(\frac{\lambda(a+c)}{a \sqrt{c}}\right)-\exp \left\{\frac{-2 \lambda^{2}}{a}\right\} \Phi\left(\frac{\lambda(c-a)}{a \sqrt{c}}\right) \\
& -\exp \left\{\frac{-2 \lambda^{2}}{b}\right\} \Phi\left(\frac{\lambda(1-b c)}{b \sqrt{c}}\right) \\
& +\exp \left\{-2 \lambda^{2}\left(\frac{1}{a}+\frac{1}{b}-2\right)\right\} \Phi\left(\frac{\lambda(1-b c-2 b)}{b \sqrt{c}}\right),
\end{aligned}
$$

where $c=a(b-1) /(b(a-1))$. 
2.2. The distribution of the maximum of the Chentsov field on polygonal lines with several linear sections. We need some results obtained in the paper [6]. Fix $n \geq 1$. Let

$$
x_{0}=0, \quad x_{n+1}=1, \quad y_{0}=1, \quad y_{n+1}=0 .
$$

Suppose a polygonal line $L$ changes its direction at each of $n$ points $Q_{1}, \ldots, Q_{n}$ with coordinates $\left(x_{1}, y_{1}\right), \ldots,\left(x_{n}, y_{n}\right)$, respectively. The line $L$ can be considered as a set of points in $D$, namely

$$
\begin{gathered}
L=\left\{(s, t): t=I_{\{0\}}(s)+\sum_{i=1}^{n+1}\left(-s k_{i}+b_{i}\right) I_{\left(x_{i-1} ; x_{i}\right]}(s), s \in[0,1]\right\}, \\
k_{i}=\frac{\left(y_{i-1}-y_{i}\right)}{x_{i}-x_{i-1}}, \quad b_{i}=\frac{x_{i} y_{i-1}-x_{i-1} y_{i}}{x_{i}-x_{i-1}}
\end{gathered}
$$

where $I_{A}$ is the indicator of the set $A$.

Let $\Delta_{0}=0$ and $\Delta_{i}=x_{i} / y_{i}, i=1, \ldots, n$.

Theorem 2.2 (Kruglova [6]). Let $\{X(s, t):(s, t) \in D\}$ be the Chentsov field defined on the unit square. Suppose the polygonal line $L$ changes its direction $n$ times at the points $Q_{1}, \ldots, Q_{n}$ with coordinates $\left(x_{1}, y_{1}\right), \ldots,\left(x_{n}, y_{n}\right)$, respectively, and let $L$ be defined by equality (5). Suppose the coordinates of these points satisfy

$$
0<x_{1}<\cdots<x_{n}<1, \quad 1>y_{1}>\cdots>y_{n}>0 .
$$

Then

$$
\begin{aligned}
P_{n}(\lambda) \stackrel{\text { def }}{=} \mathrm{P}\left\{\sup _{(s ; t) \in L} X(s ; t)<\lambda\right\} \\
=\int_{-\infty}^{\frac{\lambda}{y_{1}}} \cdots \int_{-\infty}^{\frac{\lambda}{y_{n}}}\left(1-\exp \left\{-2 \lambda\left(\frac{\lambda}{y_{n}}-u_{n}\right)\right\}\right) \\
\quad \times \prod_{i=1}^{n}\left(1-\exp \left\{-\frac{2 \lambda_{i-1} \lambda_{i}}{\left(\Delta_{i}-\Delta_{i-1}\right)}\right\}\right) \varphi_{0, \Delta_{i}-\Delta_{i-1}}\left(u_{i}-u_{i-1}\right) d u_{1} \cdots d u_{n}
\end{aligned}
$$

for all $\lambda>0$, where $\lambda_{i}=\lambda / y_{i}-u_{i}, 0 \leq i \leq n, u_{0}=0$, and

$$
\varphi_{0, \Delta}(u)=\exp \left\{-u^{2} /(2 \Delta)\right\} / \sqrt{2 \pi \Delta}
$$

is the density of the Gaussian distribution with parameters 0 and $\Delta$.

Remark 2.1. If $n=1$, then Theorem 2.2 implies that

$$
P_{1}(\lambda)=I_{1}(\lambda)-I_{2}(\lambda)-I_{3}(\lambda)+I_{4}(\lambda),
$$

where

$$
\begin{aligned}
I_{1}(\lambda) & =\int_{-\infty}^{\frac{\lambda}{y_{1}}} \varphi_{0, \Delta_{1}}\left(u_{1}\right) d u_{1}=\Phi\left(\frac{\lambda}{\sqrt{x_{1} y_{1}}}\right) \\
I_{2}(\lambda) & =\int_{-\infty}^{\frac{\lambda}{y_{1}}} \exp \left\{-\frac{2 \lambda\left(\frac{\lambda}{y_{1}}-u_{1}\right)}{\Delta_{1}}\right\} \varphi_{0, \Delta_{1}}\left(u_{1}\right) d u_{1} \\
& =\exp \left\{-\frac{2 \lambda^{2}}{x_{1}}\left(1-y_{1}\right)\right\} \Phi\left(\frac{\lambda\left(1-2 y_{1}\right)}{\sqrt{x_{1} y_{1}}}\right)
\end{aligned}
$$


and

$$
\begin{aligned}
I_{3}(\lambda) & =\int_{-\infty}^{\frac{\lambda}{y_{1}}} \exp \left\{-2 \lambda\left(\frac{\lambda}{y_{1}}-u_{1}\right)\right\} \varphi_{0, \Delta_{1}}\left(u_{1}\right) d u_{1} \\
& =\exp \left\{-\frac{2 \lambda^{2}}{y_{1}}\left(1-x_{1}\right)\right\} \Phi\left(\frac{\lambda\left(1-2 x_{1}\right)}{\sqrt{x_{1} y_{1}}}\right) \\
I_{4}(\lambda) & =\int_{-\infty}^{\frac{\lambda}{y_{1}}} \exp \left\{-2 \lambda\left(\frac{\lambda}{y_{1}}-u_{1}\right)\right\} \exp \left\{-\frac{2 \lambda\left(\frac{\lambda}{y_{1}}-u_{1}\right)}{\Delta_{1}}\right\} \varphi_{0, \Delta_{1}}\left(u_{1}\right) d u_{1} \\
& =\exp \left\{\frac{2 \lambda^{2}}{x_{1} y_{1}}\left(x_{1}+y_{1}\right)\left(x_{1}+y_{1}-1\right)\right\} \Phi\left(\frac{\lambda\left(1-2 x_{1}-2 y_{1}\right)}{\sqrt{x_{1} y_{1}}}\right) .
\end{aligned}
$$

The right hand sides of (4) and (6) coincide, since

$$
a=\frac{x_{1}}{1-y_{1}}, \quad b=\frac{y_{1}}{1-x_{1}}, \quad c=\frac{x_{1}}{y_{1}}
$$

on the right hand side of (4). This means that Theorem 2.1] is a special case of Theorem 2.2 for $n=1$.

2.3. Asymptotic behavior of the distributions of Gaussian processes. Let $Y(t)$, $t \in[0,1]$, be a zero mean Gaussian process with continuous trajectories. Suppose the variance $\sigma^{2}(t)=\mathrm{E}\left[Y^{2}(t)\right]$ attains its maximum at a unique point $t_{0}$ of the interval $[0,1]$. Denote by $\rho(s, t)$ the covariance function of the process $Y(t)$. Consider the following conditions introduced in 8 .

E1: There are constants $\beta>0$ and $A \geq 0$ such that

$$
\sigma(t)=\sigma\left(t_{0}\right)-A\left|t-t_{0}\right|^{\beta}(1+o(1)), \quad t \rightarrow t_{0} .
$$

E2: There are constants $\alpha>0$ and $C>0$ such that

$$
\rho(t, s)=1-C|t-s|^{\alpha}(1+o(1)), \quad t \rightarrow t_{0}, \quad s \rightarrow t_{0} .
$$

E3: For some $\gamma>0$ and $G$ and for all $t, s$,

$$
\mathrm{E}\left[(Y(t)-Y(s))^{2}\right] \leq G|t-s|^{\gamma} .
$$

For $0<\alpha \leq 2$, denote by $\chi(t), t \in(-\infty, \infty)$, a Gaussian process such that

1) $\mathrm{E}[\chi(t)]=-|t|^{\alpha}$ for all $t \in(-\infty, \infty)$;

2) $\operatorname{cov}[\chi(s), \chi(t)]=|t|^{\alpha}+|s|^{\alpha}-|t-s|^{\alpha}$ for all $s, t \in(-\infty, \infty)$.

For all $b>0, M<B$, and $0<\alpha<2$ let

$$
\begin{gathered}
H_{\alpha}^{b}(M, B)=\mathrm{E}\left[\exp \left\{\frac{1}{1+b} \max _{\left[(1+b)^{2 / \alpha} M,(1+b)^{2 / \alpha} B\right]} \chi(t)\right\}\right], \\
H_{\alpha}=\lim _{B \rightarrow \infty} \frac{H_{\alpha}^{0}(0, B)}{B}<\infty \\
0<H_{\alpha}^{K, 1}=\lim _{B \rightarrow \infty} H_{\alpha}^{K}(-B, B)<\infty, \quad K>0, \\
0<H_{\alpha}^{K, 2}=\lim _{B \rightarrow \infty} H_{\alpha}^{K}(0, B)<\infty .
\end{gathered}
$$

Piterbarg [8] proved that these limits are finite.

The following result is also proved in [8].

Theorem 2.3 (Piterbarg [8]). Let $Y(t), t \in[0,1]$, be a zero mean Gaussian process with continuous trajectories. Suppose its variance $\sigma^{2}(t)=\mathrm{E}\left[Y(t)^{2}\right]$ attains the maximal value $\max _{t \in T} \sigma^{2}(t)=\sigma_{M}^{2}$ at a unique point $t_{0} \in[0,1]$. Assume that conditions E1-E3 hold for $\alpha>0$. 
1) If $\alpha<\beta$, then

$\mathrm{P}\left\{\max _{t \in[0,1]} Y(t)>u\right\}= \begin{cases}2 N\left(\frac{u}{\sigma_{M}}\right)^{\frac{2}{\alpha}-\frac{2}{\beta}} \Psi\left(\frac{u}{\sigma_{M}}\right)(1+o(1)), & t_{0} \in(0,1), \\ N\left(\frac{u}{\sigma_{M}}\right)^{\frac{2}{\alpha}-\frac{2}{\beta}} \Psi\left(\frac{u}{\sigma_{M}}\right)(1+o(1)), & t_{0}=0 \text { or } t_{0}=1,\end{cases}$

as $u \rightarrow \infty$, where

$$
N=\frac{H_{\alpha} \Gamma\left(\frac{1}{\beta}\right) C^{\frac{1}{\alpha}} \sigma_{M}^{\frac{1}{\beta}}}{\beta A^{\frac{1}{\beta}}}
$$

and the constants $\beta$ and $A$ are defined in condition E1, while the constants $\alpha$ and $C$ are defined in condition E2;

2) if $\alpha=\beta$, then

$$
\mathrm{P}\left\{\max _{t \in[0,1]} Y(t)>u\right\}= \begin{cases}H_{\alpha}^{K, 1} \Psi\left(\frac{u}{\sigma_{M}}\right)(1+o(1)), & t_{0} \in(0,1), \\ H_{\alpha}^{K, 2} \Psi\left(\frac{u}{\sigma_{M}}\right)(1+o(1)), & t_{0}=1 \text { or } t_{0}=0,\end{cases}
$$

as $u \rightarrow \infty$, where $K=A /\left(C \sigma_{M}\right)$;

3) if $\alpha>\beta$, then

as $u \rightarrow \infty$.

$$
\mathrm{P}\left\{\max _{t \in[0,1]} X(t)>u\right\}=\Psi\left(\frac{u}{\sigma_{M}}\right)(1+o(1))
$$

\section{THE MAIN RESUlT}

Consider the restriction of the Chentsov field on the polygonal line with one change of direction. Let $\left(x_{1}, y_{1}\right)$ be the coordinates of the change point. Put

$$
P_{1}(\lambda)=\mathrm{P}\left\{\sup _{(s, t) \in L} X(s, t)<\lambda\right\},
$$

where $L$ is the polygonal line depicted in Figure 1 and where $\lambda>0$ is a certain constant.

The following theorem contains estimates for the distribution of the maximum of the Chentsov field on a polygonal line with one change point. The estimates depend on the position of the change point.

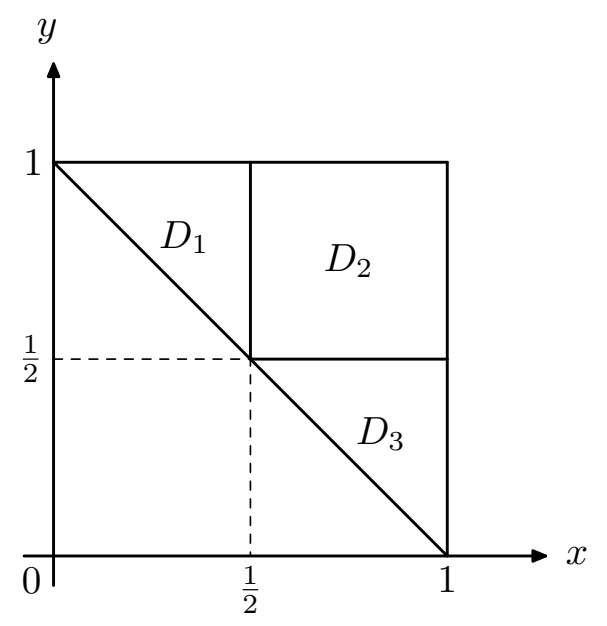

FiguRE 2. Domains where the estimates are of the same order 
Theorem 3.1. Let $X(s, t)$ be the Chentsov field defined on a unit square. Suppose a polygonal line $L$ such as depicted in Figure 1 has a unique change point $Q$ with coordinates $\left(x_{1}, y_{1}\right)$. Let

$$
\begin{aligned}
& D_{1}=\{(x, y): 0 \leq x \leq 1 / 2,1 / 2<y \leq 1, x+y>1\}, \\
& D_{2}=\{(x, y): 1 / 2<x \leq 1,1 / 2<y \leq 1\}, \\
& D_{3}=\{(x, y): 0 \leq y \leq 1 / 2,1 / 2<x \leq 1, x+y>1\}, \\
& D_{4}=\{(x, y): 0 \leq x \leq 1,0 \leq y \leq 1, x+y=1\},
\end{aligned}
$$

be the domains shown in Figure 2.

1) If $Q \in D_{1}$, then

$$
\alpha e^{-c_{1} \lambda^{2}}+e^{-c_{2} \lambda^{2}}\left(\frac{B_{1}}{\lambda}+\frac{B_{2}}{\lambda^{3}}\right)<1-P_{1}(\lambda)<e^{-c_{1} \lambda^{2}}+e^{-c_{2} \lambda^{2}}\left(\frac{B_{1}}{\lambda}+\frac{B_{3}}{\lambda^{3}}\right),
$$

where

$$
\alpha=\left\{\begin{array}{ll}
\frac{1}{2}, & x_{1}=\frac{1}{2}, \\
1, & \text { otherwise },
\end{array} \quad c_{1}=\frac{2\left(1-x_{1}\right)}{y_{1}}, \quad c_{2}=\frac{1}{2 x_{1} y_{1}},\right.
$$

and

$$
\begin{aligned}
& B_{1}= \begin{cases}\frac{\sqrt{x_{1} y_{1}}}{\sqrt{2 \pi}}\left(1+\frac{1}{2 x_{1}-1}+\frac{1}{2 y_{1}-1}-\frac{1}{2 y_{1}+2 x_{1}-1}\right), & y_{1} \neq 1 / 2, x_{1} \neq 1 / 2, \\
\frac{\sqrt{x_{1}}}{\sqrt{4 \pi}}\left(1+\frac{1}{2 x_{1}-1}-\frac{1}{2 x_{1}}\right), & y_{1}=1 / 2, x_{1} \neq 1 / 2, \\
\frac{\sqrt{y_{1}}}{\sqrt{4 \pi}}\left(1+\frac{1}{2 y_{1}-1}-\frac{1}{2 y_{1}}\right), & x_{1}=1 / 2, y_{1} \neq 1 / 2 ;\end{cases} \\
& B_{2}=-\frac{\left(x_{1} y_{1}\right)^{3 / 2}}{\sqrt{2 \pi}}\left(1+\frac{1}{\left(2 y_{1}-1\right)^{3}}\right) ; \\
& B_{3}= \begin{cases}\frac{\left(x_{1} y_{1}\right)^{3 / 2}}{\sqrt{2 \pi}}\left(\frac{1}{\left(1-2 x_{1}\right)^{3}}+\frac{1}{\left(2 x_{1}+2 y_{1}-1\right)^{3}}\right), & x_{1} \neq 1 / 2, \\
\frac{1}{32 y_{1}^{3 / 2} \sqrt{\pi}}, & x_{1}=1 / 2 .\end{cases}
\end{aligned}
$$

2) If $Q \in D_{2}$, then

$$
e^{-c_{2} \lambda^{2}}\left(\frac{B_{1}}{\lambda}+\frac{B_{4}}{\lambda^{3}}\right)<1-P_{1}(\lambda)<e^{-c_{2} \lambda^{2}}\left(\frac{B_{1}}{\lambda}+\frac{B_{5}}{\lambda^{3}}\right),
$$

where

$$
\begin{aligned}
& B_{4}=-\frac{\left(x_{1} y_{1}\right)^{3 / 2}}{\sqrt{2 \pi}}\left(1+\frac{1}{\left(2 y_{1}-1\right)^{3}}+\frac{1}{\left(2 x_{1}-1\right)^{3}}\right) ; \\
& B_{5}=-\frac{\left(x_{1} y_{1}\right)^{3 / 2}}{\sqrt{2 \pi}} \frac{1}{\left(2 x_{1}+2 y_{1}-1\right)^{3}} .
\end{aligned}
$$

3) If $Q \in D_{3}$, then

$$
\beta e^{-c_{3} \lambda^{2}}+e^{-c_{2} \lambda^{2}}\left(\frac{B_{1}}{\lambda}+\frac{B_{6}}{\lambda^{3}}\right)<1-P_{1}(\lambda)<e^{-c_{3} \lambda^{2}}+e^{-c_{2} \lambda^{2}}\left(\frac{B_{1}}{\lambda}+\frac{B_{7}}{\lambda^{3}}\right),
$$

where

$$
\beta= \begin{cases}\frac{1}{2}, & y_{1}=\frac{1}{2}, \\ 1, & \text { otherwise }\end{cases}
$$


and

$$
\begin{aligned}
B_{6} & =-\frac{\left(x_{1} y_{1}\right)^{3 / 2}}{\sqrt{2 \pi}}\left(1+\frac{1}{\left(2 x_{1}-1\right)^{3}}\right) ; \\
B_{7} & = \begin{cases}\frac{\left(x_{1} y_{1}\right)^{3 / 2}}{\sqrt{2 \pi}}\left(\frac{1}{\left(2 y_{1}-1\right)^{3}}+\frac{1}{\left(2 x_{1}+2 y_{1}-1\right)^{3}}\right), & y_{1} \neq 1 / 2, \\
\frac{1}{32 \sqrt{\pi} x_{1}^{3 / 2}}, & y_{1}=1 / 2 ;\end{cases}
\end{aligned}
$$

and $c_{3}=2\left(1-y_{1}\right) / x_{1}$.

4) If $Q \in D_{4}$, then

$$
1-P_{1}(\lambda)=e^{-2 \lambda^{2}}
$$

Remark 3.1. All coefficients $c_{1}, c_{2}, c_{3}$, and $B_{1}, \ldots, B_{7}$ depend on the coordinates of the point $Q$.

Proof. First we consider the case of $Q \in D_{4}$. Substituting $x_{1}=1-y_{1}$ in ([6), we obtain

$$
\begin{aligned}
P_{1}(\lambda)= & \Phi\left(\frac{\lambda}{\sqrt{\left(1-y_{1}\right) y_{1}}}\right)-e^{-2 \lambda^{2}} \Phi\left(\frac{\lambda\left(1-2 y_{1}\right)}{\sqrt{\left(1-y_{1}\right) y_{1}}}\right) \\
& -e^{-2 \lambda^{2} \Phi} \Phi\left(\frac{\lambda\left(2 y_{1}-1\right)}{\sqrt{\left(1-y_{1}\right) y_{1}}}\right)+\Phi\left(-\frac{\lambda}{\sqrt{\left(1-y_{1}\right) y_{1}}}\right) \\
= & 1-e^{-2 \lambda^{2}} .
\end{aligned}
$$

In what follows we apply the inequalities

$$
\frac{e^{-x^{2} / 2}}{\sqrt{2 \pi}}\left(\frac{1}{x}-\frac{1}{x^{3}}\right)<1-\Phi(x)<\frac{e^{-x^{2} / 2}}{x \sqrt{2 \pi}}, \quad x>0 .
$$

Since $1-\Phi(x)=\Phi(-x), x>0$, an analogous inequality holds for $\Phi(-x), x>0$, as well. For the first term in (6), we have

$$
\frac{\exp \left\{-\lambda^{2} /\left(2 x_{1} y_{1}\right)\right\} \sqrt{x_{1} y_{1}}}{\lambda \sqrt{2 \pi}}\left(1-\frac{x_{1} y_{1}}{\lambda^{2}}\right)<1-I_{1}(\lambda)<\frac{\exp \left\{-\lambda^{2} /\left(2 x_{1} y_{1}\right)\right\} \sqrt{x_{1} y_{1}}}{\lambda \sqrt{2 \pi}} .
$$

Now we represent $I_{2}(\lambda)$ as follows:

$$
I_{2}(\lambda)=\exp \left\{-\frac{2 \lambda^{2}}{x_{1}}\left(1-y_{1}\right)\right\}-\exp \left\{-\frac{2 \lambda^{2}}{x_{1}}\left(1-y_{1}\right)\right\}\left(1-\Phi\left(\frac{\lambda\left(1-2 y_{1}\right)}{\sqrt{x_{1} y_{1}}}\right)\right) .
$$

If $y_{1}<1 / 2$, then

$$
\begin{gathered}
I_{2}(\lambda)>\exp \left\{-\frac{2 \lambda^{2}}{x_{1}}\left(1-y_{1}\right)\right\}-\frac{\exp \left\{-\lambda^{2} /\left(2 x_{1} y_{1}\right)\right\} \sqrt{x_{1} y_{1}}}{\lambda \sqrt{2 \pi}\left(1-2 y_{1}\right)}, \\
I_{2}(\lambda)<\exp \left\{-\frac{2 \lambda^{2}}{x_{1}}\left(1-y_{1}\right)\right\}-\frac{\exp \left\{-\lambda^{2} /\left(2 x_{1} y_{1}\right)\right\} \sqrt{x_{1} y_{1}}}{\lambda \sqrt{2 \pi}\left(1-2 y_{1}\right)}\left(1-\frac{x_{1} y_{1}}{\left(1-2 y_{1}\right)^{2} \lambda^{2}}\right) .
\end{gathered}
$$

If $y_{1}>1 / 2$, then

$$
\frac{\exp \left\{-\lambda^{2} /\left(2 x_{1} y_{1}\right)\right\} \sqrt{x_{1} y_{1}}}{\lambda \sqrt{2 \pi}\left(2 y_{1}-1\right)}\left(1-\frac{x_{1} y_{1}}{\left(2 y_{1}-1\right)^{2} \lambda^{2}}\right)<I_{2}(\lambda)<\frac{\exp \left\{-\lambda^{2} /\left(2 x_{1} y_{1}\right)\right\} \sqrt{x_{1} y_{1}}}{\lambda \sqrt{2 \pi}\left(2 y_{1}-1\right)},
$$

and if $y_{1}=1 / 2$, then

$$
I_{2}(\lambda)=\frac{1}{2} e^{-\lambda^{2} / x_{1}}
$$

We reduce $I_{3}(\lambda)$ to the following form:

$$
I_{3}(\lambda)=\exp \left\{-\frac{2 \lambda^{2}}{y_{1}}\left(1-x_{1}\right)\right\}-\exp \left\{-\frac{2 \lambda^{2}}{y_{1}}\left(1-x_{1}\right)\right\}\left(1-\Phi\left(\frac{\lambda\left(1-2 x_{1}\right)}{\sqrt{x_{1} y_{1}}}\right)\right) .
$$


If $x_{1}<1 / 2$, then

$$
\begin{gathered}
I_{3}(\lambda)>\exp \left\{-\frac{2 \lambda^{2}}{y_{1}}\left(1-x_{1}\right)\right\}-\frac{\exp \left\{-\lambda^{2} /\left(2 x_{1} y_{1}\right)\right\} \sqrt{x_{1} y_{1}}}{\lambda \sqrt{2 \pi}\left(1-2 x_{1}\right)}, \\
I_{3}(\lambda)<\exp \left\{-\frac{2 \lambda^{2}}{y_{1}}\left(1-x_{1}\right)\right\}-\frac{\exp \left\{-\lambda^{2} /\left(2 x_{1} y_{1}\right)\right\} \sqrt{x_{1} y_{1}}}{\lambda \sqrt{2 \pi}\left(1-2 x_{1}\right)}\left(1-\frac{x_{1} y_{1}}{\left(1-2 x_{1}\right)^{2} \lambda^{2}}\right) .
\end{gathered}
$$

If $x_{1}>1 / 2$, then

$$
\frac{\exp \left\{-\lambda^{2} /\left(2 x_{1} y_{1}\right)\right\} \sqrt{x_{1} y_{1}}}{\lambda \sqrt{2 \pi}\left(2 x_{1}-1\right)}\left(1-\frac{x_{1} y_{1}}{\left(2 x_{1}-1\right)^{2} \lambda^{2}}\right)<I_{3}(\lambda)<\frac{\exp \left\{-\lambda^{2} /\left(2 x_{1} y_{1}\right)\right\} \sqrt{x_{1} y_{1}}}{\lambda \sqrt{2 \pi}\left(2 x_{1}-1\right)},
$$

and if $x_{1}=1 / 2$, then

$$
I_{3}(\lambda)=\frac{1}{2} e^{-\lambda^{2} / y_{1}}
$$

Finally

$$
\begin{gathered}
I_{4}(\lambda)<\frac{\exp \left\{-\lambda^{2} /\left(2 x_{1} y_{1}\right)\right\} \sqrt{x_{1} y_{1}}}{\lambda \sqrt{2 \pi}\left(2 x_{1}+2 y_{1}-1\right)} \\
I_{4}>\frac{\exp \left\{-\lambda^{2} /\left(2 x_{1} y_{1}\right)\right\} \sqrt{x_{1} y_{1}}}{\lambda \sqrt{2 \pi}\left(2 x_{1}+2 y_{1}-1\right)}\left(1-\frac{x_{1} y_{1}}{\left(2 x_{1}+2 y_{1}-1\right)^{2} \lambda^{2}}\right) .
\end{gathered}
$$

Combining the inequalities obtained above and using representation (6), we complete the proof of the theorem.

The following corollary explains the dependence of the asymptotic behavior of the maximum of the Chentsov field on a polygonal line with a unique change point on the position of this point.

Corollary 3.1. $\quad$ 1. If $Q \in D_{1}$, then

$$
1-P_{1}(\lambda)=e^{-c_{1} \lambda^{2}}+\frac{B_{1} e^{-c_{2} \lambda^{2}}}{\lambda}+o\left(\frac{e^{-c_{2} \lambda^{2}}}{\lambda}\right) \quad \text { as } \lambda \rightarrow \infty .
$$

2. If $Q \in D_{2}$, then

$$
1-P_{1}(\lambda)=\frac{B_{1} e^{-c_{2} \lambda^{2}}}{\lambda}+o\left(\frac{e^{-c_{2} \lambda^{2}}}{\lambda}\right) \quad \text { as } \lambda \rightarrow \infty .
$$

3. If $Q \in D_{3}$, then

$$
1-P_{1}(\lambda)=e^{-c_{3} \lambda^{2}}+\frac{B_{1} e^{-c_{2} \lambda^{2}}}{\lambda}+o\left(\frac{e^{-c_{2} \lambda^{2}}}{\lambda}\right) \quad \text { as } \lambda \rightarrow \infty .
$$

4. If $Q \in D_{4}$, then

$$
1-P_{1}(\lambda)=e^{-2 \lambda^{2}}
$$

where the constants $B_{1}, c_{1}, c_{2}$, and $c_{3}$ are defined in Theorem 3.1 .

Remark 3.2. To separate the main term in the asymptotic formula, we find the relations between the coefficients in the estimates for the tail of the distribution of the maximum of the Chentsov field defined on a polygonal line. Namely,

1) if $Q \in D_{1}$, then $c_{1} \leq c_{2} ;$ moreover, $c_{1}=c_{2}$ only if $x_{1}=\frac{1}{2}$;

2) if $Q \in D_{3}$, then $c_{3} \leq c_{2}$; moreover, $c_{3}=c_{2}$ only if $y_{1}=\frac{1}{2}$.

Therefore, the main term of the asymptotics in (8) and (9) is determined by the first summand. 


\section{Corollary 3.2.}

$$
\begin{aligned}
& \text { 1) Let } Q \in D_{1} \text {. If } x_{1} \neq \frac{1}{2} \text {, then } \\
& \lim _{\lambda \rightarrow \infty} \lambda e^{c_{2} \lambda^{2}}\left(1-P_{1}(\lambda)-e^{-c_{1} \lambda^{2}}\right)=B_{1},
\end{aligned}
$$

while if $x_{1}=\frac{1}{2}$, then

$$
\lim _{\lambda \rightarrow \infty} e^{c_{2} \lambda^{2}}\left(1-P_{1}(\lambda)\right)=\frac{1}{2}
$$

2) Let $Q \in D_{2}$. Then

$$
\lim _{\lambda \rightarrow \infty} \lambda e^{c_{2} \lambda^{2}}\left(1-P_{1}(\lambda)\right)=B_{1}
$$

3) Let $Q \in D_{3}$. If $y_{1} \neq \frac{1}{2}$, then

$$
\lim _{\lambda \rightarrow \infty} \lambda e^{c_{2} \lambda^{2}}\left(1-P_{1}(\lambda)-e^{-c_{3} \lambda^{2}}\right)=B_{1},
$$

while if $y_{1}=\frac{1}{2}$, then

$$
\lim _{\lambda \rightarrow \infty} \lambda e^{c_{2} \lambda^{2}}\left(1-P_{1}(\lambda)\right)=\frac{1}{2} .
$$

Remark 3.3. We want to determine the "maximal" and "minimal" asymptotics. First we define these notions.

Let $D$ be a set of parameters, $f_{Q}(\cdot)$ a family of functions, $Q \in D$, and $g(\cdot)$ a fixed function. We write

$$
\max _{Q \in D} f_{Q}(\lambda)=g(\lambda)
$$

if, for all $Q \in D$, either $f_{Q}(\lambda)=g(\lambda)$ or $\lim _{\lambda \rightarrow \infty} f_{Q}(\lambda) / g(\lambda)=0$.

Similarly we write

$$
\min _{Q \in D} f_{Q}(\lambda)=g(\lambda)
$$

if, for all $Q \in D$, either $f_{Q}(\lambda)=g(\lambda)$ or $\lim _{\lambda \rightarrow \infty} g(\lambda) / f_{Q}(\lambda)=0$.

We show how the order of the asymptotic formulas for $1-P_{1}(\lambda)$ changes in different domains, namely

$\mathbf{D}_{1}: \min _{D_{1}}\left(1-P_{1}(\lambda)\right)=e^{-2 \lambda^{2}} ; \max _{D_{1}}\left(1-P_{1}(\lambda)\right)=e^{-\lambda^{2}}$.

$\mathbf{D}_{\mathbf{3}}: \min _{D_{3}}\left(1-P_{1}(\lambda)\right)=e^{-2 \lambda^{2}} ; \max _{D_{3}}\left(1-P_{1}(\lambda)\right)=e^{-\lambda^{2}}$.

$\mathbf{D}_{\mathbf{2}}: \min _{D_{2}}\left(1-P_{1}(\lambda)\right)=e^{-2 \lambda^{2}} ; \max _{D_{2}}\left(1-P_{1}(\lambda)\right)=\frac{2^{5 / 2}}{3 \sqrt{\pi}} \lambda^{-1} e^{-\lambda^{2} / 2}$.

The maximal asymptotics for the whole square $D$ is

$$
\max _{D}\left(1-P_{1}(\lambda)\right)=\frac{2^{5 / 2}}{3 \sqrt{\pi}} \lambda^{-1} e^{-\lambda^{2} / 2} .
$$

This result can be used to obtain a lower estimate of the tail of the distribution of the maximum of the Chentsov field on the unit square:

$$
\mathrm{P}\left\{\max _{(s, t) \in D} X(s, t)>\lambda\right\}>\frac{2^{5 / 2}}{3 \sqrt{\pi}} \lambda^{-1} e^{-\lambda^{2} / 2} .
$$

The following result is a generalization of Theorem 3.1 .

Theorem 3.2. Let $X(s, t)$ be the Chentsov field defined on the unit square. Suppose a polygonal line $L$ has $n$ change points $Q_{1}, \ldots, Q_{n}$ whose coordinates $\left(x_{1}, y_{1}\right), \ldots,\left(x_{n}, y_{n}\right)$ 
satisfy the assumptions of Theorem [2.2, Put $x_{i}=a_{i} x_{1}$ and $y_{i}=b_{i} y_{1}, i=2, \ldots, n$. Consider the following domains in $R^{2 n}$ :

$$
\begin{aligned}
& D_{1}=\left\{y_{1}>1 / 2, a_{i} b_{i}<1, \frac{a_{i} b_{i-1}-a_{i-1} b_{i}}{2\left(b_{i-1}-b_{i}\right)}>a_{i}\right. \\
& \text { or } \left.a_{i-1}>\frac{a_{i} b_{i-1}-a_{i-1} b_{i}}{2\left(b_{i-1}-b_{i}\right)}, a_{i} x_{1}+b_{i} y_{1} \geq 1, i=2, \ldots, n, a_{n} x_{1} \geq 1 / 2\right\}, \\
& D_{j}=\left\{y_{1}>1 / 2, a_{j} b_{j}>1, a_{i} b_{i}<1, \frac{a_{i} b_{i-1}-a_{i-1} b_{i}}{2\left(b_{i-1}-b_{i}\right)}>a_{i}\right. \\
& \text { or } \left.a_{i-1}>\frac{a_{i} b_{i-1}-a_{i-1} b_{i}}{2\left(b_{i-1}-b_{i}\right)}, a_{i} x_{1}+b_{i} y_{1} \geq 1, i=2, \ldots, n, i \neq j, a_{n} x_{1} \geq 1 / 2\right\} \text {, } \\
& j=2, \ldots, n, \\
& D_{n+j}=\left\{y_{1}>1 / 2, a_{j-1}<\frac{a_{j} b_{j-1}-a_{j-1} b_{j}}{2\left(b_{j-1}-b_{j}\right)}<a_{j}, j \neq i, \frac{a_{i} b_{i-1}-a_{i-1} b_{i}}{2\left(b_{i-1}-b_{i}\right)}>a_{i}\right. \\
& \text { or } \left.a_{i-1}>\frac{a_{i} b_{i-1}-a_{i-1} b_{i}}{2\left(b_{i-1}-b_{i}\right)}, a_{i} x_{1}+b_{i} y_{1} \geq 1, i=2, \ldots, n, a_{n} x_{1} \geq 1 / 2\right\} \text {, } \\
& D_{n+1}=\left\{y_{1} \leq 1 / 2, a_{i} b_{i}<1, \frac{a_{i} b_{i-1}-a_{i-1} b_{i}}{2\left(b_{i-1}-b_{i}\right)}>a_{i}\right. \\
& \text { or } \left.a_{i-1}>\frac{a_{i} b_{i-1}-a_{i-1} b_{i}}{2\left(b_{i-1}-b_{i}\right)}, a_{i} x_{1}+b_{i} y_{1} \geq 1, i=2, \ldots, n, a_{n} x_{1} \geq 1 / 2\right\} \\
& D_{2 n+1}=\left\{a_{n} x_{1}<1 / 2, a_{i} x_{1}+b_{i} y_{1} \geq 1, i=2, \ldots, n\right\} \text {. }
\end{aligned}
$$

Let

$$
K_{1}=2 y_{1}-1, \quad K_{j}=\frac{2\left(x_{j-1} y_{j}+x_{j} y_{j-1}-2 x_{j} y_{j}\right)}{\left(x_{j} y_{j-1}-x_{j-1} y_{j}\right)}, \quad j=2, \ldots, n \text {. }
$$

$\operatorname{Set}(\vec{x}, \vec{y})=\left(x_{1}, \ldots, x_{n}, y_{1}, \ldots, y_{n}\right)$. Then

$$
1-P_{n}(\lambda)= \begin{cases}H_{1}^{K_{j}, 1} \Psi\left(\frac{\lambda}{\sqrt{x_{j} y_{j}}}\right)(1+o(1)), & (\vec{x}, \vec{y}) \in D_{j}, j=1, \ldots, n, \\ \exp \left\{-\frac{2\left(1-y_{1}\right) \lambda^{2}}{x_{1}}\right\}(1+o(1)), & (\vec{x}, \vec{y}) \in D_{n+1}, \\ \exp \left\{-\frac{2\left(b_{j-1}-b_{j}\right)\left(a_{j}-a_{j-1}\right) \lambda^{2}}{\left(a_{j} b_{j-1}-a_{j-1} b_{j}\right)^{2} x_{1} y_{1}}\right\}(1+o(1)), & (\vec{x}, \vec{y}) \in D_{j+n}, j=2, \ldots, n, \\ \exp \left\{-\frac{2\left(1-x_{n}\right) \lambda^{2}}{y_{n}}\right\}(1+o(1)), & (\vec{x}, \vec{y}) \in D_{2 n+1}\end{cases}
$$

as $\lambda \rightarrow \infty$.

Proof. Let the polygonal line $L$ be given by (5). Then the covariance function of the restriction of the Chentsov field to this polygonal line is equal to

$$
\begin{aligned}
r(s, t)=s( & \sum_{i=2}^{n}\left(-\frac{\left(y_{i-1}-y_{i}\right) t}{x_{i}-x_{i-1}}+\frac{x_{i} y_{i-1}-x_{i-1} y_{i}}{x_{i}-x_{i-1}}\right) I_{\left(x_{i-1}, x_{i}\right]}(t) \\
& \left.+\left(-\frac{\left(1-y_{1}\right) t}{x_{1}}+1\right) I_{\left[0, x_{1}\right]}(t)+\left(-\frac{y_{n} t}{1-x_{n}}+\frac{y_{n}}{1-x_{n}}\right) I_{\left(x_{n}, 1\right]}(t)\right), \quad s \leq t .
\end{aligned}
$$


Thus the variance $\sigma^{2}$ of this process is given by

$$
\begin{aligned}
g(t)= & \sigma^{2}(t) \\
=t\left(\sum_{i=2}^{n}\right. & \left(-\frac{\left(y_{i-1}-y_{i}\right) t}{x_{i}-x_{i-1}}+\frac{x_{i} y_{i-1}-x_{i-1} y_{i}}{x_{i}-x_{i-1}}\right) I_{\left(x_{i-1}, x_{i}\right]}(t) \\
& \left.\quad+\left(1-\frac{\left(1-y_{1}\right) t}{x_{1}}\right) I_{\left[0, x_{1}\right]}(t)+\left(-\frac{y_{n} t}{1-x_{n}}+\frac{y_{n}}{1-x_{n}}\right) I_{\left(x_{n}, 1\right]}(t)\right) .
\end{aligned}
$$

Now we find the maximum of the variance between the change points.

Case 1. If $t \in\left[0, x_{1}\right]$, then

$$
g(t)=t-\frac{\left(1-y_{1}\right) t^{2}}{x_{1}}=\frac{\left(1-y_{1}\right)}{x_{1}}\left(t-\frac{x_{1}}{2\left(1-y_{1}\right)}\right)^{2}+\frac{x_{1}}{4\left(1-y_{1}\right)} .
$$

If $x_{1} /\left(2\left(1-y_{1}\right)\right)<x_{1}$, then

$$
\max _{t \in\left[0, x_{1}\right]} g(t)=\frac{x_{1}}{4\left(1-y_{1}\right)} .
$$

Otherwise, the function $g$ increases in the interval $\left[0, x_{1}\right]$, and one needs to evaluate the function $g$ at the point $x_{1}$ :

$$
g\left(x_{1}\right)=x_{1} y_{1}
$$

Thus

$$
\max _{t \in\left[0, x_{1}\right]} g(t)= \begin{cases}\frac{x_{1}}{4\left(1-y_{1}\right)}, & y_{1} \leq 1 / 2 \\ x_{1} y_{1}, & y_{1}>1 / 2 .\end{cases}
$$

Case 2. If $t \in\left(x_{i-1}, x_{i}\right], i=2, \ldots, n$, then

$$
\begin{aligned}
g(t) & =-\frac{\left(y_{i-1}-y_{i}\right) t^{2}}{x_{i}-x_{i-1}}+\frac{\left(x_{i} y_{i-1}-x_{i-1} y_{i}\right) t}{x_{i}-x_{i-1}} \\
& =-\frac{\left(y_{i-1}-y_{i}\right)}{x_{i}-x_{i-1}}\left(t-\frac{\left(x_{i} y_{i-1}-x_{i-1} y_{i}\right)}{2\left(y_{i}-y_{i-1}\right)}\right)^{2}+\frac{\left(x_{i} y_{i-1}-x_{i-1} y_{i}\right)^{2}}{4\left(x_{i}-x_{i-1}\right)\left(y_{i}-y_{i-1}\right)} .
\end{aligned}
$$

If

$$
\frac{\left(x_{i} y_{i-1}-x_{i-1} y_{i}\right)}{2\left(y_{i}-y_{i-1}\right)} \in\left(x_{i-1}, x_{i}\right]
$$

then

$$
\max _{t \in\left(x_{i-1}, x_{i}\right]} g(t)=\frac{\left(x_{i} y_{i-1}-x_{i-1} y_{i}\right)^{2}}{4\left(x_{i}-x_{i-1}\right)\left(y_{i}-y_{i-1}\right)} .
$$

Otherwise, one needs to find the values at the endpoints of the interval:

$$
g\left(x_{i-1}\right)=x_{i-1} y_{i-1}, \quad g\left(x_{i}\right)=x_{i} y_{i} .
$$

Comparing these values, we conclude that

$$
\max _{t \in\left(x_{i-1}, x_{i}\right]} g(t)= \begin{cases}a_{i-1} b_{i-1} x_{1} y_{1}, & a_{i} b_{i}<1, A_{i}>a_{i} \text { or } a_{i-1}>A_{i}, \\ \frac{x_{1} y_{1}\left(a_{i} b_{i-1}-a_{i-1} b_{i}\right)^{2}}{4\left(a_{i}-a_{i-1}\right)\left(b_{i-1}-b_{i}\right)}, & a_{i-1}<A_{i}<a_{i}, \\ a_{i} b_{i} x_{1} y_{1}, & a_{i} b_{i}>1, A_{i}>a_{i} \text { or } a_{i-1}>A_{i},\end{cases}
$$

where

$$
A_{i}=\frac{a_{i} b_{i-1}-a_{i-1} b_{i}}{2\left(b_{i-1}-b_{i}\right)}
$$


Case 3. If $t \in\left(x_{n}, 1\right]$, then

$$
g(t)=-\frac{y_{n} t}{1-x_{n}}(1-t)=-\frac{y_{n}}{1-x_{n}}\left(t-\frac{1}{2}\right)^{2}+\frac{y_{n}}{4\left(1-x_{n}\right)} .
$$

If $\frac{1}{2} \in\left(x_{n}, 1\right]$, then

$$
\max _{t \in\left(x_{n}, 1\right]} g(t)=\frac{y_{n}}{4\left(1-x_{n}\right)} .
$$

Otherwise the function $g$ decreases in the interval $\left(x_{n}, 1\right]$, and one needs to evaluate the function $g$ at the point $x_{n}$ :

$$
g\left(x_{n}\right)=x_{n} y_{n}=a_{n} b_{n} x_{1} y_{1} .
$$

Now one can determine the maximum of the variance:

$$
\max _{t \in\left(x_{n}, 1\right]} g(t)= \begin{cases}\frac{b_{n} y_{1}}{4\left(1-a_{n} x_{1}\right)}, & a_{n} x_{1}<1 / 2 . \\ a_{n} b_{n} x_{1} y_{1}, & a_{n} x_{1} \geq 1 / 2,\end{cases}
$$

The case of $\left(x_{1}, \ldots, x_{n}, y_{1}, \ldots, y_{n}\right) \in D_{1}$. In this case,

$$
\sigma(t)=\sqrt{t\left(1-\frac{\left(1-y_{1}\right) t}{x_{1}}\right)},
$$

and the maximum of the function $g$ is attained at the point $x_{1}$, that is,

$$
\max _{t \in[0,1]} g(t)=g\left(x_{1}\right)=x_{1} y_{1} \text {. }
$$

Thus

whence

$$
\sigma_{M}=\sqrt{x_{1} y_{1}}
$$

$$
\sigma(t)=\sqrt{x_{1} y_{1}}\left(1-\frac{\left(2 y_{1}-1\right)\left(t-x_{1}\right)}{2 x_{1} y_{1}}\right)(o(1)+1), \quad t \rightarrow x_{1} .
$$

The covariance function is of the form

$$
\rho(s, t)=\sqrt{\frac{s\left(1-\frac{\left(1-y_{1}\right) t}{x_{1}}\right)}{t\left(1-\frac{\left(1-y_{1}\right) s}{x_{1}}\right)} .}
$$

Moreover,

$$
\rho(s, t)=1-\frac{1}{2 x_{1} y_{1}}(t-s)(1+o(1)), \quad s, t \rightarrow x_{1} .
$$

Using conditions E1 and E2 and representations (10) and (11), we find that

$$
\alpha=\beta=1, \quad A=\frac{\left(2 y_{1}-1\right)}{2 \sqrt{x_{1} y_{1}}}, \quad C=\frac{1}{2 x_{1} y_{1}}, \quad \sigma_{M}=\sqrt{x_{1} y_{1}}, \quad K_{1}=2 y_{1}-1 .
$$

Applying Theorem 2.3 (case 2), we obtain

$$
\mathrm{P}\left\{\max _{L} X(s, t)>u\right\}=H_{1}^{K, 1} \Psi\left(\frac{u}{\sqrt{x_{1} y_{1}}}\right)(1+o(1)) .
$$

The case of $\left(x_{1}, \ldots, x_{n}, y_{1}, \ldots, y_{n}\right) \in D_{n+1}$. In this case,

$$
\sigma(t)=\sqrt{t\left(1-\frac{\left(1-y_{1}\right) t}{x_{1}}\right)}
$$

and the maximum of the function $g$ is attained at the point $x_{1} /\left(2\left(1-y_{1}\right)\right)$, that is,

$$
\max _{t \in[0,1]} g(t)=g\left(\frac{x_{1}}{2\left(1-y_{1}\right)}\right)=\frac{x_{1}}{4\left(1-y_{1}\right)} .
$$


Therefore

$$
\sigma_{M}=\sqrt{\frac{x_{1}}{4\left(1-y_{1}\right)}}
$$

whence

$$
\sigma(t)=\left(\sqrt{\frac{x_{1}}{4\left(1-y_{1}\right)}}-\left(\frac{1-y_{1}}{x_{1}}\right)^{3 / 2}\left(t-\frac{x_{1}}{2\left(1-y_{1}\right)}\right)^{2}\right)(o(1)+1) .
$$

The covariance function can be written as follows:

$$
\rho(s, t)=\left(1-\frac{2\left(1-y_{1}\right)}{x_{1}}(t-s)\right)(1+o(1)), \quad s, t \rightarrow \frac{x_{1}}{2\left(1-y_{1}\right)} .
$$

Now we obtain from (12) and (13) that

$$
\alpha=1, \quad \beta=2, \quad A=\left(\frac{1-y_{1}}{x_{1}}\right)^{3 / 2}, \quad C=\frac{2\left(1-y_{1}\right)}{x_{1}} .
$$

Applying Theorem 2.3, 1), we get

$$
\mathrm{P}\left\{\max _{(s, t) \in L} X(s, t)>u\right\}=\exp \left\{-\frac{2\left(1-y_{1}\right) u^{2}}{x_{1}}\right\}(1+o(1)) .
$$

The case of $\left(x_{1}, \ldots, x_{n}, y_{1}, \ldots, y_{n}\right) \in D_{n+j}, j=2, \ldots, n$. In this case,

$$
\sigma(t)=\sqrt{t\left(-\frac{\left(y_{j-1}-y_{j}\right) t}{x_{j}-x_{j-1}}+\frac{x_{j} y_{j-1}-x_{j-1} y_{j}}{x_{j}-x_{j-1}}\right)} .
$$

The maximum of the function $g$ is attained at the point

$$
\frac{\left(a_{j} b_{j-1}-a_{j-1} b_{j}\right) x_{1}}{2\left(b_{j-1}-b_{j}\right)},
$$

that is,

$$
\max _{t \in[0,1]} g(t)=g\left(\frac{\left(a_{j} b_{j-1}-a_{j-1} b_{j}\right) x_{1}}{2\left(b_{j-1}-b_{j}\right)}\right)=\frac{x_{1} y_{1}\left(a_{j} b_{j-1}-a_{j-1} b_{j}\right)^{2}}{4\left(a_{j}-a_{j-1}\right)\left(b_{j-1}-b_{j}\right)} .
$$

Hence

$$
\begin{gathered}
\sigma_{M}=\sqrt{\frac{x_{1} y_{1}\left(a_{j} b_{j-1}-a_{j-1} b_{j}\right)^{2}}{4\left(a_{j}-a_{j-1}\right)\left(b_{j-1}-b_{j}\right)}}, \\
\sigma(t)=\left(\sqrt{\frac{x_{1} y_{1}\left(a_{j} b_{j-1}-a_{j-1} b_{j}\right)^{2}}{4\left(a_{j}-a_{j-1}\right)\left(b_{j-1}-b_{j}\right)}}\right. \\
-\frac{\left(b_{j-1}-b_{j}\right) y_{1} \sqrt{\left(a_{j}-a_{j-1}\right)\left(b_{j-1}-b_{j}\right)}}{x_{1}\left(a_{j}-a_{j-1}\right)\left(a_{j} b_{j-1}-a_{j-1} b_{j}\right) \sqrt{x_{1} y_{1}}} \\
\left.\times\left(t-\frac{\left(a_{j} b_{j-1}-a_{j-1} b_{j}\right) x_{1}}{2\left(b_{j-1}-b_{j}\right)}\right)^{2}\right)(o(1)+1) .
\end{gathered}
$$

The covariance function can be written as follows:

$$
\rho(s, t)=\left(1-\frac{2\left(b_{j-1}-b_{j}\right)}{x_{1}\left(a_{j} b_{j-1}-a_{j-1} b_{j}\right)}(t-s)\right)(1+o(1)) .
$$

Then we obtain from (14) and (15) that $\alpha=1, \beta=2$, and

$$
A=\frac{\left(b_{j-1}-b_{j}\right) y_{1} \sqrt{\left(a_{j}-a_{j-1}\right)\left(b_{j-1}-b_{j}\right)}}{x_{1}\left(a_{j}-a_{j-1}\right)\left(a_{j} b_{j-1}-a_{j-1} b_{j}\right) \sqrt{x_{1} y_{1}}}, \quad C=\frac{2\left(b_{j-1}-b_{j}\right)}{x_{1}\left(a_{j} b_{j-1}-a_{j-1} b_{j}\right)} .
$$


Applying Theorem 2.3, we get

$$
\mathrm{P}\left\{\max _{L} X(s, t)>u\right\}=\exp \left\{-\frac{2\left(a_{j}-a_{j-1}\right)\left(b_{j-1}-b_{j}\right) u^{2}}{x_{1} y_{1}\left(a_{j} b_{j-1}-a_{j-1} b_{j}\right)^{2}}\right\}(1+o(1)) .
$$

The case of $\left(x_{1}, \ldots, x_{n}, y_{1}, \ldots, y_{n}\right) \in D_{j}, j=2, \ldots, n$. In this case,

$$
\max _{t \in[0,1]} g(t)=g\left(x_{j}\right)=x_{j} y_{j}=a_{j} b_{j} x_{1} y_{1} .
$$

Since

$$
\begin{aligned}
\sigma(t)= & \left(\sqrt{x_{j} y_{j}}-\frac{\left(x_{j-1} y_{j}+x_{j} y_{j-1}-2 x_{j} y_{j}\right)}{\left(x_{j}-x_{j-1}\right) \sqrt{x_{j} y_{j}}}\left(t-x_{j}\right)\right)\left(1+o\left(\left|t-x_{j}\right|\right)\right), \\
& \rho(s, t)=\left(1-\frac{\left(x_{j} y_{j-1}-x_{j-1} y_{j}\right)}{2\left(x_{j}-x_{j-1}\right) x_{j} y_{j}}(t-s)\right)(1+o(|t-s|)),
\end{aligned}
$$

we obtain $\alpha=1, \beta=1$, and

$$
\begin{gathered}
A=\frac{\left(x_{j-1} y_{j}+x_{j} y_{j-1}-2 x_{j} y_{j}\right)}{\left(x_{j}-x_{j-1}\right) \sqrt{x_{j} y_{j}}}, \quad C=\frac{\left(x_{j} y_{j-1}-x_{j-1} y_{j}\right)}{2\left(x_{j}-x_{j-1}\right) x_{j} y_{j}}, \\
K_{j}=\frac{2\left(x_{j-1} y_{j}+x_{j} y_{j-1}-2 x_{j} y_{j}\right)}{\left(x_{j} y_{j-1}-x_{j-1} y_{j}\right)}
\end{gathered}
$$

Therefore

$$
\mathrm{P}\left\{\max _{(s, t) \in L} X(s, t)>u\right\}=H_{1}^{K_{j}, 1} \Psi\left(\frac{u}{\sqrt{x_{j} y_{j}}}\right)(1+o(1)), \quad j=2, \ldots, n .
$$

The case of $\left(x_{1}, \ldots, x_{n}, y_{1} \ldots, y_{n}\right) \in D_{2 n+1}$. We have

$$
\max _{t \in[0,1]} g(t)=g(1 / 2)=\frac{b_{n} y_{1}}{4\left(1-a_{n} x_{1}\right)}
$$

and

$$
\mathrm{P}\left\{\max _{(s, t) \in L} X(s, t)>u\right\}=\exp \left\{-\frac{2\left(1-a_{n} x_{1}\right) u^{2}}{b_{n} y_{1}}\right\}(1+o(1)) .
$$

\section{Concluding Remarks}

In this paper, we have obtained estimates for the tail of the distribution of the maximum of the Chentsov field on a polygonal line with a unique change point. We showed how the estimates depend on the position of the change point. We have also found the behavior of the tail of the distribution of the maximum of the field $X(s, t)$ on polygonal lines with several change points.

\section{BIBLIOGRAPHY}

1. N. N. Chentsov, Wiener random fields of several parameters, Dokl. Akad. Nauk SSSR 46 (1956), no. 4, 607-609. (Russian)

2. E. Csaki, D. Khoshnevisan, and Z. Shi, Boundary crossings and the distribution function of the maximum of Brownian sheet, Stoch. Process. Appl. 90 (2000), no. 1, 1-18. MR.1787122 (2001m:60115)

3. V. Goodman, Distribution estimates for functionals of the two-parameter Wiener process, Ann. Probab. 4 (1976), no. 6, 977-982. MR0423556 (54:11532)

4. I. I. Klesov, On the probability of attainment of a curvilinear level by a Wiener field, Teor. Ĭmovir. Mat. Stat. 51 (1994), 62-66; English transl. in Theory Probab. Math. Statist. 51 (1995), 63-67. MR1445053 (97m:60074)

5. O. I. Klesov and N. V. Kruglova, The distribution of functionals such as the maximum for a two-parameter Chentsov field, Naukovi Visti NTUU (KPI) 2007, no. 4, 136-141. (Ukrainian)

6. N. V. Kruglova, Distribution of the maximum of the Chentsov random field, Theor. Stoch. Process. 14 (2008), no. 1, 76-81. MR 2479708 (2009m:60119) 
7. S. R. Paranjape and C. Park, Distribution of the supremum of the two-parameter Yeh-Wiener process on the boundary, Appl. Probab. 10 (1973), no. 4, 875-880. MR0381015 (52:1912)

8. V. I. Piterbarg, Gaussian random processes, Probability theory. Mathematical statistics. Theoretical cybernetics, Akad. Nauk SSSR, Vsesoyuz. Inst. Nauchn. i Tekhn. Informatsii (1982), no. 19, 155-199. MR657960 (84h:60077)

9. J. Yeh, Wiener measure in a space of functions of two variables, Trans. Amer. Math. Soc. (1960), no. 95, 433-450. MR0125433 (23:A2735)

Department of Mathematical Analysis and Probability Theory, Faculty for Physics and Mathematics, National Technical University of Ukraine (KPI), Peremogy Avenue 37, Kyiv 03056, UKRAINE

E-mail address: natahak@ukr.net

Received 22/SEP/2009

Translated by S. KVASKO 